\title{
MORPHOMETRIC ANALYSIS OF Coptotermes sp. POPULATION FROM TWO DIFFERENT NESTS
}

\author{
Analisis Morfometik Populasi Coptotermes sp. Dari Dua Sarang yang Berbeda \\ Astuti Arif, Ira Taskirawati, and Affandi
}

\begin{abstract}
The aim of this research is to study morphmetric variation of external anatomy of subterranean termite (Coptotermes sp.). The samples were collected from two different nests, i.e. Laboratory of Forest Product Engineering and Diversification, Hasanuddin University (site A) and Kompleks Perumahan Dosen Unhas, Tamalanrea (site B). Resulted data were statistically analyzed for mean, standard deviation, standard error and coefficient of variability. The mean values of the different population samples were compared by using student't' test according to SPSS Ver.12. For each individual soldier and worker, 54 variables of external characters were measured. The result showed that some external characters of soldier and worker casts were significantly different with different locations. In soldier casts, eleven of 54 variables were found to be significant different in length and width of caput, length and width metathorax, mesothorax length, length and width of abdomens, scape length, flagellum width, femur width, and width tibia. Whilst, in worker casts, 11 of 54 variables were found to be significant different in mesothorax length, scape length, flagellum width, trochanter width, tibia length, tarsus length, and claw width. However, morph metric of external characters of Coptotermes sp. at two locations was generally relative same in terms of coefficient of variability.
\end{abstract}

Key words: Morphometric analysis, Coptotermes sp.

\section{INTRODUCTION}

Basically, the balance of the ecosystem can occur because of the close interaction between living things and the environment. Forest, for example, produces environmental conditions (microclimate) which strongly support the development of things living in it, including bodymicroorganism or microorganisms on and in the ground. Meanwhile, microorganism helps to decompose dead organisms such as litter, plants and animals. The produced nutrient elements are needed by the forest vegetation to grow well. Microorganisms such as termites, mold, bacteria, and other have very important roles in maintaining the stability of this ecosystem.

In an ecosystem, termites have a very important role, namely as decomposer for litter and dead vegetation. Termites (ordo: Isoptera) has high diversity on ecosystem. In the world, it had been found about 2,000 species, which consist of 7 families, 15 sub-families, and 200 genera; while in Indonesia, it had been found about $10 \%$ of the total termite of world or around
200 species, including 3 families, 6 subfamily and 14 genera. However, about five percent of the 200 species found in Indonesia are harmful to humans, namely as a pest for most agricultural and plantation crops (Tarumingkeng, 2001).

One of termites types intensively investigated by researchers is subterranean termite. It is well known because of its building nest in the soil, but it can reach the food source by creating a tunnel to keep the humidity. In addition, it can attack without directly contact to the ground as long as they get moisture continuously. Genus Coptotermes (Family Rhinotermitidae) is very destructive pest to wood and wooden material in the world (Takematsu et al, 2000) and various species of the termite are found in Indonesia, such as in Java, Sulawesi and Sumatra. Taketmatsu et.al. (2006) also mentions that Coptotermes found in South Sulawesi had different characteristics to Coptotermes curvignatus found in Java and those had been regarded as the same species. Nevertheless, the information about that termites is very limited. The other side, the damage caused by them is very seriously 
dangerous and can be found in various habitat conditions, such as resident, government and private buildings. Therefore, data and information on all aspects associated with this termite, including morphometric of external anatomy, are needed to explore in order to the precisely control and prevent its damage. This study was aimed at analyzing the morphometric variations of Coptotermes sp. from various locations. The results of this study is expected to contribute to the development of science, by providing data for comparative studies of Coptotermes in the future.

\section{MATERIALS AND METHODS}

\section{Specimen preparation}

The soldiers and workers of Coptotemes sp. were collected from the areas of the Engineering and Diversification of Forest Products Laboratory, Forestry Faculty, Hasanuddin University (Site A), and The Resident of Hasanuddin University, Tamalanrea, Makassar, South Sulawesi, Indonesia (Site B). Specimen was prepared in alcohol solution $70 \%$ prior to laboratory analysis.

\section{Variable Observations}

All specimens were observed with a binocular microscope equipped with a variety of magnification and a calibrated micrometer. The external anatomy of specimens including 54 variabels, i.e., body (2), head without eyes (2) , abdomen (2), antenna (6), leg (36), and thorax (6) width, were measured.

\section{Data Analysis}

Measurement data of morphometric parameters, such as average, standard deviation, range and coefficient of variance were descriptively analyzed. Student ' $\mathrm{t}$ ' test was conducted to know the different site effects against morphometric respons.

\section{RESULTS AND DISCUSSION}

\section{Workers}

The results of measurements and statistical analysis of workers morphometrics at two locations can be shown at Appendix 1. The
Student test indicated that almost all external characters of termites from both locations were not significantly different. There were only 7 (seven) body parts of workers found varied beetwen the two locations, namely thorax (mesothoraks length), antenna (scape length and width of the flagellum), legs: the front legs (trochanter width), the middle legs (tibial width and length of tarsus) and the rear legs (width claw). The general characteristics of Coptotermes sp. morphometric (Appendix 2.) indicated that the variation of morphometric parameters were relatively similar in term of coefficient of variability $(<30 \%)$.

Thorax consists of three segments, namely prothorax, mesothorax, and metathorax. Thorax has a dorsal appearence (notum), ventral appearence (sternum), and lateral side (pleura). The different length of mesothoraks between site $A$ and site $B$ can be used for taxonomic purposes. According to Nandika et al. (2003), the dorsal appearence of thorax varied in form and can be used for taxonomic purposes.

Antenna is the sensor tools for insects in detecting environmental changes, supporting insect activities, and looking for their pairs. Termite's antenna has miniliform shape. The first segment (scape) was relatively long. The second segment or pedicel was generally shorter than scape. Other segments behind pedicel formed like-flagellum. The flagellum length was different between site $A$ and site $B$ presumbly because of the influence of environmental factors, such as temperature and humidity which can influence the sensitivity level of termites in stimulating of sound or vibration form. Nandika et al. (2003) stated that the third segment had large variations among termite species, which can be used as a taxonomic characteristic of proliferation on youth development.

Legs can also become a taxonomic identification tool for insects. Legs consist of several segments, namely: coxa, trochanter, femur, tibia and tarsus. In the last segment (tarsus) was generally equipped with claws. Variation of leg size suppose due to alleged differences in the feeding and cruising activities of termites. Besides for walking, legs were also used for scratching foods. 
Soldier

The results of measurements and statistical analysis of workers morphometrics at two locations can be shown at Appendix 3. The Student test indicated that almost all external characters of termites from both locations were not significantly different. There were only 11 (eleven) body parts of soldiers found varied beetwen the two locations, namely head (lenght and width of caput), thorax (mesothorax length, lenght and width metathorax), antenna (scape length and flagellum width), abdomen (abdominal length and width); legs: the front legs (femur width) and the rear legs (width tibia). The general characteristics of Coptotermes sp. morphometric (Appendix 4.) indicated that the variation of external characters were relatively similar in term of coefficient of variability $(<30 \%)$.

The head capsule of reproductive and workers were oval or slightly rounded, while soldiers were often oval and even larger (Nandika et al., 2003). The length and width of caput were found to be significant different between both sites. It indicated that there are environmental factors affecting its variation of head (caput) size. Nandika et al. (2003) stated that the head capsule form of soldier was greatly vary and very helpful for termite identification. The head capsule of soldiers had a long form to joint muscles for mandible (Harris, 1971).

The lenght and width of abdomen were showed to be significant difference between the two nests. According to Nandika et al. (2003), the soldier abdomen was smaller than worker because the main function of worker is digesting cellulose and giving it to nymphs, soldiers, and reproductives. The soldier legs were also had the same functions as the workers. But for the soldier legs, there is a noticeable difference on the front legs (femur width) and the rear legs (tibia width).

\section{CONCLUSIONS}

Morphometric variation of Coptotermes sp. specimens collected from different nests was relatively similar in term of coefficient of variance, eventhough some variabels such as thorax (mesothoraks length), antenna (scape length and width of the flagellum), legs: the front legs (trochanter width), the middle legs (tibial width and length of tarsus ) and the rear legs (width claw) of workers; and head (lenght and width of caput), thorax (mesothorax length, lenght and width metathorax), antenna (scape length and flagellum width), abdomen (abdominal length and width); legs: the front legs (femur width) and the rear legs (width tibia) of soldiers were found to be significantly different.

\section{REFERENCES}

Harris, W.V. 1971. Termites: Their Recognition and Control. $2^{\text {nd }}$ edition. Longmans. London.

Nandika, D., Y. Rismayadi, dan F. Diba. 2003. Rayap: Biologi dan Pengendaliannya. Muhammadiyyah Univ. Press. Surakarta.

Takematsu, Y., T. Yoshimura, S.Yusuf, Y. Yanase, K.Kambara, A. Tashiro, S. Doi, M. Takeshi, P. Sukartana, T. Inoue, H. Yuzawa, M. Ohkuma, T. Kudo, Y.Sornnuwat, and C. Vongkaluang. 2006. termite Assemlages in Urban Areas of South East Asia: Diversity and Economic Impacts. In: Sustainable Development and Utilization of Tropical Forest Resources (Ed: Y. Imamura et.al.). Report of JSPS-LIPI Core University Program in the Field of Wood Science 1995-2006. Kyoto. Japan. pp: 84-91.

Takematsu, Y., T. Yoshimura, M. Takahashi, S.Yusuf, and P. Sukartana. 2000. Present Status of an Important Pest Termite Genus, Coptotermes, in Indonesia. In: Sustainable Utilization of Forest Products: SocioEconomical and Ecological Management of Tropical Forests. Proceeding of the Third International Wood Science Symposium. JSPS-LIPI Core University Program in the Field of Wood Science 1995-2006. Kyoto. Japan. pp: 161-166.

Tarumingkeng, R. 2001. Biologi dan Prilaku Rayap. Pusat Studi IImu Hayat. Institut Pertanian Bogor. Bogor.

Weesner, F.M. 1969. External Anatomy. In: Biology of Termite. Vol II. Krisna, K. and F. M. Weesner (Ed.) Academic Press. New York. 
Diterima : 22 Februari 2010

Astuti Arif, Ira Taskirawati, and Affandi

Lab. Pemanfaatan dan Pengolahan Hasil Hutan

Department of Forestry, Forestry Faculty

Hasanuddin University - Indonesia

e-mail: astuti_arif@yahoo.com 
Appendix 1. Student Test Analysis of Coptotermes sp. Worker Morphometric in Site A and B.

\begin{tabular}{|c|c|c|c|c|c|c|c|}
\hline \multirow{2}{*}{ No } & \multirow{2}{*}{ Variable } & \multicolumn{2}{|c|}{ Mean } & \multirow{2}{*}{$\begin{array}{c}\text { Mean } \\
\text { difference }\end{array}$} & \multicolumn{2}{|c|}{$95 \%$ Confidence interval } & \multirow{2}{*}{ Remarks } \\
\hline & & Site A & Site $B$ & & Lower bound & Upper bound & \\
\hline 1 & Body weight (gram) & 0.00407 & 0.00395 & 0.00012 & -0.00029 & 0.00053 & tn \\
\hline 2 & Body lenght (mm) & 4.8055 & 5.005 & -0.1995 & -0.337 & -0.062 & tn \\
\hline \multicolumn{8}{|c|}{ Caput (mm) } \\
\hline 1 & Caput length & 0.79 & 0.8375 & -0.0475 & -0.079 & -0.016 & tn \\
\hline 2 & Caput width & 1.3875 & 1.5375 & -0.15 & -0.199 & -0.101 & tn \\
\hline \multicolumn{8}{|c|}{ Thoraks (mm) } \\
\hline 1 & Prothorax length & 0.395 & 0.348 & 0.047 & 0.0301 & 0.064 & tn \\
\hline 2 & Prothorax width & 0.5425 & 0.65 & -0.1075 & -0.136 & -0.079 & tn \\
\hline 3 & Metathorax length & 0.316 & 0.336 & -0.02 & -0.046 & -0.006 & tn \\
\hline 4 & Metathorax width & 0.6525 & 0.7075 & -0.055 & -0.091 & -0.019 & tn \\
\hline 5 & Mesothorax length & 0.362 & 0.421 & -0.059 & -0.081 & -0.037 & * \\
\hline 6 & Mesothorax width & 0.8825 & 1.05 & -0.0775 & -0.239 & -0.096 & tn \\
\hline \multicolumn{8}{|c|}{ Abdomen (mm) } \\
\hline 1 & Abdomen length & 2.9425 & 3.0625 & -0.12 & -0.203 & 0.113 & tn \\
\hline 2 & Abdomen width & 1.405 & 1.495 & -0.09 & -0.146 & -0.034 & tn \\
\hline \multicolumn{8}{|c|}{ Antena (mm) } \\
\hline 1 & Scape length & 0.15 & 0.144 & 0.006 & -0.657 & 0.208 & * \\
\hline 2 & Scape width & 0.082 & 0.066 & 0.016 & 0.0116 & 0.02 & tn \\
\hline 3 & Pedicel length & 0.099 & 0.062 & 0.037 & 0.0298 & 0.044 & tn \\
\hline 4 & Pedicel width & 0.073 & 0.066 & 0.007 & 0.0014 & 0.013 & tn \\
\hline 5 & Flagellum length & 1.2475 & 1.07 & 0.1775 & 0.1382 & 0.216 & tn \\
\hline 6 & Flagellum width & 0.098 & 0.092 & 0.006 & -0.12 & 0.013 & ${ }^{*}$ \\
\hline \multicolumn{8}{|c|}{ Front legs (mm) } \\
\hline 1 & Coxa length & 0.319 & 0.311 & 0.008 & -0.013 & 0.029 & tn \\
\hline 2 & Coxa width & 0.204 & 0.205 & -0.001 & -0.021 & 0.019 & tn \\
\hline 3 & Trochanter length & 0.109 & 0.092 & 0.017 & 0.0001 & 0.034 & tn \\
\hline 4 & Trochanter width & 0.084 & 0.079 & 0.005 & 0.0039 & 0.014 & * \\
\hline 5 & Femur length & 0.754 & 0.705 & 0.049 & 0.0162 & 0.082 & tn \\
\hline 6 & Femur width & 0.208 & 0.187 & 0.021 & 0.009 & 0.033 & tn \\
\hline 7 & Tibia length & 0.684 & 0.6855 & -0.0015 & -0.039 & 0.036 & tn \\
\hline 8 & Tibia width & 0.128 & 0.126 & 0.002 & -0.016 & 0.020 & tn \\
\hline 9 & Tarsus length & 0.272 & 0.264 & 0.008 & -0.014 & 0.030 & tn \\
\hline 10 & Tarsus width & 0.055 & 0.056 & -0.001 & -0.007 & 0.005 & tn \\
\hline 11 & Claw length & 0.055 & 0.048 & 0.007 & 0.0007 & 0.013 & tn \\
\hline 12 & Claw width & 0.023 & 0.025 & -0.002 & -0.007 & 0.003 & tn \\
\hline \multicolumn{8}{|c|}{ Middle legs $(\mathrm{mm})$} \\
\hline 1 & Coxa length & 0.258 & 0.286 & -0.028 & -0.084 & 0.028 & tn \\
\hline 2 & Coxa width & 0.117 & 0.119 & -0.002 & -0.019 & 0.015 & tn \\
\hline 3 & Trochanter length & 0.111 & 0.126 & -0.015 & -0.03 & 0.0003 & tn \\
\hline 4 & Trochanter width & 0.058 & 0.065 & -0.007 & -0.016 & 0.002 & tn \\
\hline 5 & Femur length & 0.676 & 0.674 & 0.002 & -0.024 & 0.028 & tn \\
\hline 6 & Femur width & 0.141 & 0.148 & -0.007 & -0.028 & 0.014 & tn \\
\hline 7 & Tibia length & 0.66 & 0.671 & -0.011 & -0.033 & 0.0011 & tn \\
\hline 8 & Tibia width & 0.123 & 0.111 & 0.012 & -0.005 & 0.029 & * \\
\hline 9 & Tarsus length & 0.247 & 0.253 & -0.006 & -0.037 & 0.025 & * \\
\hline 10 & Tarsus width & 0.057 & 0.06 & -0.003 & -0.01 & 0.004 & tn \\
\hline 11 & Claw length & 0.058 & 0.06 & -0.002 & -0.01 & 0.006 & tn \\
\hline 12 & Claw width & 0.024 & 0.023 & 0.001 & -0.004 & 0.006 & tn \\
\hline
\end{tabular}


Appendix 1. To be continued

\begin{tabular}{|c|c|c|c|c|c|c|c|}
\hline \multirow{2}{*}{ No } & \multirow{2}{*}{ Variable } & \multicolumn{2}{|c|}{ Mean } & \multirow{2}{*}{$\begin{array}{c}\text { Mean } \\
\text { difference }\end{array}$} & \multicolumn{2}{|c|}{$95 \%$ confidence interval } & \multirow{2}{*}{ Remarks } \\
\hline & & Site $A$ & Site $B$ & & Lower bound & Upper bound & \\
\hline \multicolumn{8}{|c|}{ Rear legs (mm) } \\
\hline 1 & Coxa length & 0.246 & 0.225 & 0.021 & -0.001 & 0.043 & tn \\
\hline 2 & Coxa width & 0.098 & 0.087 & 0.011 & -0.022 & 0.028 & tn \\
\hline 3 & Trochanter length & 0.102 & 0.084 & 0.018 & 0.0045 & 0.031 & tn \\
\hline 4 & Trochanter width & 0.061 & 0.067 & -0.006 & -0.017 & 0.005 & tn \\
\hline 5 & Femur length & 0.688 & 0.727 & -0.039 & -0.064 & -0.014 & tn \\
\hline 6 & Femur width & 0.194 & 0.168 & 0.026 & 0.003 & 0.049 & tn \\
\hline 7 & Tibia length & 0.894 & 0.817 & 0.077 & 0.0566 & 0.097 & tn \\
\hline 8 & Tibia width & 0.113 & 0.119 & -0.006 & -0.02 & 0.008 & tn \\
\hline 9 & Tarsus length & 0.281 & 0.269 & 0.012 & -0.007 & 0.031 & tn \\
\hline 10 & Tarsus width & 0.063 & 0.069 & -0.006 & -0.017 & 0.005 & tn \\
\hline 11 & Claw length & 0.1 & 0.09 & 0.01 & 0.0052 & 0.031 & tn \\
\hline 12 & Claw width & 0.025 & 0.022 & 0.003 & -0.001 & 0.007 & * \\
\hline
\end{tabular}

Remarks: * signifficant difference; tn - no significant difference

Appendix 2. General characteristics of Coptotermes sp.) worker morphometrics in site A and B.

\begin{tabular}{|c|c|c|c|c|c|}
\hline No & Parameters & O.R & Mean \pm SD & C.V $(\%)$ & $S^{2}$ \\
\hline 1 & Body weigth & $0.0034-0.0048$ & $0.0040 \pm 0.0004$ & 10.67 & 0.00000018 \\
\hline 2 & Body length & $4.535-5.125$ & $4.9058 \pm 0.1697$ & 3.46 & 0.028803 \\
\hline 3 & Caput length & $1.3-1.6$ & $1.4625 \pm 0.0920$ & 6.29 & 0.008454 \\
\hline 4 & Caput width & $0.725-0.875$ & $0.8138 \pm 0.0409$ & 5.039 & 0.001676 \\
\hline 5 & Prothorax length & $0.32-0.42$ & $0.3715 \pm 0.0298$ & 3.66 & 0.000887 \\
\hline 6 & Prothorax width & $0.5-0.7$ & $0.5963 \pm 0.0625$ & 10.47 & 0.004153 \\
\hline 7 & Metathorax length & $0.27-0.38$ & $0.3260 \pm 0.0286$ & 8.76 & 0.000815 \\
\hline 8 & Metathorax width & $0.625-0.750$ & $0.6800 \pm 0.0470$ & 6.91 & 0.002211 \\
\hline 9 & Mesothorax length & $0.32-0.44$ & $0.3915 \pm 0.0379$ & 9.67 & 0.001434 \\
\hline 10 & Mesothorax width & $0.75-1.175$ & $0.9663 \pm 0.1136$ & 11.76 & 0.012913 \\
\hline 11 & Abdomen length & $2.85-3.175$ & $3.0025 \pm 0.1057$ & 3.52 & 0.011178 \\
\hline 12 & Abdomen width & $1.25-1.575$ & $1.4500 \pm 0.0743$ & 5.13 & 0.005526 \\
\hline 13 & Scape length & $0.14-0.16$ & $0.1470 \pm 0.0066$ & 4.47 & 0.000043 \\
\hline 14 & Scape width & $0.06-0.09$ & $0.0740 \pm 0.0094$ & 12.71 & 0.000088 \\
\hline 15 & Pedicel length & $0.05-0.11$ & $0.0805 \pm 0.0204$ & 25.32 & 0.000416 \\
\hline 16 & Pedicel width & $0.06-0.08$ & $0.0695 \pm 0.0069$ & 9.88 & 0.000047 \\
\hline 17 & Flagellum length & $1.000-1.275$ & $1.1588 \pm 0.0998$ & 8.61 & 0.009952 \\
\hline 18 & Flagellum width & $0.08-0.10$ & $0.0950 \pm 0.0061$ & 6.39 & 0.000037 \\
\hline \multirow[t]{13}{*}{19} & Front legs & & & & \\
\hline & Coxa length & $0.280-0.375$ & $0.3150 \pm 0.0224$ & 7.12 & 0.000503 \\
\hline & Coxa width & $0.17-0.24$ & $0.2045 \pm 0.0204$ & 9.98 & 0.000416 \\
\hline & Trochanter length & $0.075-0.130$ & $0.1005 \pm 0.0195$ & 19.42 & 0.000381 \\
\hline & Trochanter width & $0.07-0.10$ & $0.0815 \pm 0.0096$ & 11.79 & 0.000092 \\
\hline & Femur length & $0.65-0.80$ & $0.7295 \pm 0.0422$ & 5.79 & 0.001784 \\
\hline & Femur width & $0.17-0.23$ & $0.1975 \pm 0.0164$ & 8.31 & 0.000270 \\
\hline & Tibia length & $0.625-0.750$ & $0.6848 \pm 0.0386$ & 5.63 & 0.001488 \\
\hline & Tibia width & $0.10-0.16$ & $0.1270 \pm 0.0187$ & 14.70 & 0.000348 \\
\hline & Tarsus length & $0.22-0.30$ & $0.2680 \pm 0.0231$ & 8.61 & 0.000533 \\
\hline & Tarsus width & $0.05-0.07$ & $0.0555 \pm 0.0061$ & 10.90 & 0.000037 \\
\hline & Claw length & $0.04-0.06$ & $0.0515 \pm 0.0075$ & 14.47 & 0.000056 \\
\hline & Claw width & $0.02-0.03$ & $0.0240 \pm 0.0050$ & 20.94 & 0.000025 \\
\hline \multirow[t]{3}{*}{20} & Middle legs & & & & \\
\hline & Coxa length & $0.28-0.32$ & $0.2720 \pm 0.0596$ & 21.92 & 0.003554 \\
\hline & Coxa width & $0.12-0.15$ & $0.1180 \pm 0.0177$ & 14.96 & 0.000312 \\
\hline
\end{tabular}


Appendix 2. to be continued

\begin{tabular}{|l|l|c|c|c|c|}
\hline No & \multicolumn{1}{|c|}{ Parameters } & O.R & Mean \pm SD & C.V $(\%)$ & $S^{2}$ \\
\hline & Trochanter length & $0.10-0.15$ & $0.1185 \pm 0.0755$ & 14.81 & 0.000308 \\
\hline & Trochanter width & $0.05-0.08$ & $0.0615 \pm 0.0099$ & 16.07 & 0.000098 \\
\hline & Femur length & $0.63-0.72$ & $0.6750 \pm 0.0274$ & 4.06 & 0.000753 \\
\hline & Femur width & $0.10-0.18$ & $0.1445 \pm 0.0216$ & 14.97 & 0.000468 \\
\hline & Tibia length & $0.62-0.70$ & $0.6655 \pm 0.0231$ & 3.46 & 0.000531 \\
\hline & Tibia width & $0.09-0.15$ & $0.1170 \pm 0.019$ & 16.19 & 0.000359 \\
\hline & Tarsus length & $0.2-0.3$ & $0.2500 \pm 0.0323$ & 12.91 & 0.001042 \\
\hline & Tarsus width & $0.05-0.07$ & $0.0585 \pm 0.0075$ & 12.74 & 0.000056 \\
\hline & Claw length & $0.05-0.07$ & $0.0590 \pm 0.0079$ & 13.36 & 0.000062 \\
\hline & Claw width & $0.02-0.03$ & $0.0235 \pm 0.0049$ & 20.82 & 0.000024 \\
\hline 21 & Rear legs & & & & 10.71 \\
\hline & Coxa length & $0.2-0.3$ & $0.2355 \pm 0.0252$ & 17.14 & 0.000637 \\
\hline & Coxa width & $0.07-0.14$ & $0.0925 \pm 0.0159$ & 17.82 & 0.000251 \\
\hline & Trochanter length & $0.07-0.13$ & $0.0930 \pm 0.0166$ & 17.85 & 0.000131 \\
\hline & Trochanter width & $0.05-0.09$ & $0.0640 \pm 0.0114$ & 4.58 & 0.001051 \\
\hline & Femur length & $0.65-0.77$ & $0.7075 \pm 0.0324$ & 15.09 & 0.000746 \\
\hline & Femur width & $0.13-0.22$ & $0.1810 \pm 0.0273$ & 5.23 & 0.002005 \\
\hline & Tibia length & $0.79-0.92$ & $0.8555 \pm 0.0448$ & 12.63 & 0.000215 \\
\hline & Tibia width & $0.10-0.14$ & $0.1160 \pm 0.0147$ & 7.60 & 0.000437 \\
\hline & Tarsus length & $0.24-0.31$ & $0.2750 \pm 0.0209$ & 17.31 & 0.000131 \\
\hline & Tarsus width & $0.05-0.08$ & $0.0660 \pm 0.0114$ & 17.79 & 0.000262 \\
\hline & Claw length & $0.06-0.12$ & $0.0910 \pm 0.0162$ & 20.82 & 0.000024 \\
\hline & Claw width & $0.02-0.03$ & $0.0235 \pm 0.0049$ & &
\end{tabular}

Appendix 3. Student Test Analysis of Coptotermes sp. Soldier Morphometric in Site A and B.

\begin{tabular}{|c|c|c|c|c|c|c|c|}
\hline \multirow{2}{*}{$\begin{array}{l}\mathrm{N} \\
0\end{array}$} & \multirow{2}{*}{ Parameters } & \multicolumn{2}{|c|}{ Mean } & \multirow{2}{*}{$\begin{array}{c}\text { Mean } \\
\text { difference }\end{array}$} & \multicolumn{2}{|c|}{$95 \%$ confidence intervals } & \multirow{2}{*}{ Remarks } \\
\hline & & Site A & Site B & & Lower bound & Upper bound & \\
\hline 1 & Body weight (gram) & 0.003 & 0.003 & -0.00021 & -0.00056 & 0.0001385 & $\operatorname{tn}$ \\
\hline 2 & Body length (mm) & 5.233 & 5.638 & -0.405 & -0.70042 & 0.5042039 & $\operatorname{tn}$ \\
\hline \multicolumn{8}{|c|}{ Caput } \\
\hline 1 & Caput length & 1.95 & 2.015 & -0.065 & -0.11646 & -0.013534 & * \\
\hline 2 & Caput width & 0.978 & 0.913 & 0.065 & -0.0079 & 0.1379043 & * \\
\hline 3 & Mandible length & 0.905 & 0.973 & -0.0675 & -0.11265 & -0.022352 & $\operatorname{tn}$ \\
\hline 4 & Mandible width & 0.285 & 0.265 & 0.02 & -0.01266 & 0.0526602 & tn \\
\hline \multicolumn{8}{|c|}{ Thorax } \\
\hline 1 & Prothorax length & 0.403 & 0.425 & -0.022 & -0.04237 & -0.00163 & tn \\
\hline 2 & Prothorax width & 0.77 & 0.725 & -0.1075 & 0.014879 & 0.0751214 & tn \\
\hline 3 & Metathorax length & 0.343 & 0.302 & 0.0405 & 0.009321 & 0.7167941 & * \\
\hline 4 & Metathorax width & 0.725 & 0.692 & 0.033 & -0.02806 & 0.0940554 & * \\
\hline 5 & Mesothorax length & 0.339 & 0.313 & 0.026 & 0.000952 & 0.0089519 & * \\
\hline 6 & Mesothorax width & 0.788 & 0.845 & -0.0575 & -0.09167 & -0.023326 & tn \\
\hline \multicolumn{8}{|c|}{ Abdomen } \\
\hline 1 & Abdomen length & 2.32 & 2.63 & -0.31 & -0.43615 & -0.183847 & * \\
\hline 2 & Abdomen width & 1.203 & 1.258 & -0.055 & -0.11933 & 0.0093273 & * \\
\hline \multicolumn{8}{|c|}{ Antennae } \\
\hline 1 & Scape length & 0.156 & 0.11 & 0.0455 & 0.20521 & 0.070479 & * \\
\hline 2 & Scape width & 0.083 & 0.073 & 0.0095 & -0.00151 & 0.0205118 & tn \\
\hline 3 & Pedicel length & 0.084 & 0.075 & 0.0085 & -0.00451 & 0.0215123 & $\operatorname{tn}$ \\
\hline 4 & Pedicel width & 0.061 & 0.064 & -0.003 & -0.01044 & 0.0044444 & tn \\
\hline 5 & Flagellum length & 1.28 & 1.265 & 0.015 & -0.06446 & 0.0944625 & * \\
\hline 6 & Flagellum width & 0.098 & 0.079 & 0.019 & 0.000952 & 0.0089519 & tn \\
\hline
\end{tabular}


Appendix 3. To be continued

\begin{tabular}{|c|c|c|c|c|c|c|c|}
\hline $\begin{array}{l}\mathrm{N} \\
0\end{array}$ & Parameters & \multicolumn{2}{|c|}{ Mean } & $\begin{array}{c}\text { Mean } \\
\text { difference }\end{array}$ & \multicolumn{2}{|c|}{$95 \%$ confidence intervals } & Remarks \\
\hline \multicolumn{8}{|c|}{ Front Legs } \\
\hline 1 & Coxa length & 0.293 & 0.301 & -0.008 & -0.0211 & 0.0051016 & tn \\
\hline 2 & Coxa width & 0.18 & 0.178 & 0.002 & -0.01005 & 0.0140485 & $\operatorname{tn}$ \\
\hline 3 & Trochanter length & 0.158 & 0.171 & -0.013 & -0.02253 & -0.003475 & tn \\
\hline 4 & Trochanter width & 0.061 & 0.062 & -0.001 & -0.01 & 0.0079956 & tn \\
\hline 5 & Femur length & 0.809 & 0.758 & 0.051 & 0.029245 & 0.0727547 & tn \\
\hline 6 & Femur width & 0.216 & 0.232 & -0.016 & -0.03102 & -0.00098 & * \\
\hline 7 & Tibia length & 0.766 & 0.744 & 0.022 & 0.005606 & 0.0383977 & $\operatorname{tn}$ \\
\hline 8 & Tibia width & 0.122 & 0.146 & -0.024 & -0.04144 & -0.006562 & tn \\
\hline 9 & Tarsus length & 0.236 & 0.286 & -0.05 & 0.066394 & -0.033606 & $\operatorname{tn}$ \\
\hline 10 & Tarsus width & 0.053 & 0.056 & -0.003 & 0.007698 & 0.0016978 & $\operatorname{tn}$ \\
\hline 11 & Claw length & 0.106 & 0.103 & 0.003 & -0.00483 & 0.0108297 & tn \\
\hline 12 & Claw width & 0.027 & 0.028 & -0.001 & -0.00526 & 0.0032598 & tn \\
\hline \multicolumn{8}{|c|}{ Middle legs } \\
\hline 1 & Coxa length & 0.305 & 0.304 & 0.001 & -0.01064 & 0.0146444 & tn \\
\hline 2 & Coxa width & 0.19 & 0.183 & 0.007 & -0.00899 & 0.0022985 & $\operatorname{tn}$ \\
\hline 3 & Trochanter length & 0.178 & 0.2 & -0.022 & -0.03851 & -0.005487 & tn \\
\hline 4 & Trochanter width & 0.07 & 0.079 & -0.009 & -0.02049 & 0.0024859 & $\operatorname{tn}$ \\
\hline 5 & Femur length & 0.775 & 0.699 & 0.076 & 0.053264 & 0.0987357 & tn \\
\hline 6 & Femur width & 0.193 & 0.206 & -0.013 & -0.0281 & 0.0021013 & tn \\
\hline 7 & Tibia length & 0.722 & 0.716 & 0.006 & -0.01101 & 0.0250049 & $\operatorname{tn}$ \\
\hline 8 & Tibia width & 0.109 & 0.104 & 0.005 & -0.00614 & 0.0161391 & $\operatorname{tn}$ \\
\hline 9 & Tarsus length & 0.248 & 0.243 & 0.005 & -0.05786 & 0.057813 & $\operatorname{tn}$ \\
\hline 10 & Tarsus width & 0.054 & 0.062 & -0.008 & -0.01343 & -0.002575 & tn \\
\hline 11 & Length Claw & 0.101 & 0.105 & -0.004 & -0.01601 & 0.008777 & tn \\
\hline \multirow[t]{2}{*}{12} & Width claw & 0.024 & 0.025 & -0.001 & -0.0059 & 0.0039022 & tn \\
\hline & & Site A & Site $B$ & & Lower bound & Upper bound & \\
\hline \multicolumn{8}{|c|}{ Rear legs } \\
\hline 1 & Coxa length & 0.309 & 0.302 & 0.007 & -0.0044 & 0.0184002 & tn \\
\hline 2 & Coxa width & 0.204 & 0.198 & 0.006 & -0.00653 & 0.0185275 & tn \\
\hline 3 & Trochanter length & 0.183 & 0.192 & -0.009 & -0.02107 & 0.0030689 & tn \\
\hline 4 & Trochanter width & 0.069 & 0.057 & 0.012 & 0.004015 & 0.0199847 & $\operatorname{tn}$ \\
\hline 5 & Femur length & 0.924 & 0.896 & 0.028 & 0.009105 & 0.0468953 & $\operatorname{tn}$ \\
\hline 6 & Femur width & 0.238 & 0.216 & 0.022 & 0.006341 & 0.0376594 & tn \\
\hline 7 & Tibia length & 0.946 & 0.931 & 0.015 & 0.011878 & 0.0418776 & $\operatorname{tn}$ \\
\hline 8 & Tibia width & 0.144 & 0.108 & 0.036 & 0.02387 & 0.0481297 & * \\
\hline 9 & Tarsus length & 0.294 & 0.304 & -0.01 & -0.02415 & 0.0041455 & tn \\
\hline 10 & Tarsus width & 0.058 & 0.051 & 0.007 & 0.001354 & 0.0126461 & tn \\
\hline 11 & Claw length & 0.113 & 0.135 & -0.022 & -0.03464 & -0.009356 & tn \\
\hline 12 & Claw width & 0.026 & 0.025 & 0.001 & -0.0039 & 0.0059022 & tn \\
\hline
\end{tabular}

Remarks: * signifficant difference; tn - no significant difference 
Appendix 4. General characteristics of Coptotermes sp. workers morphometric in site A and B.

\begin{tabular}{|c|c|c|c|c|c|}
\hline No & Parameters & O.R & Mean $\pm S D$ & C.V $(\%)$ & $S^{2}$ \\
\hline 1 & Body weight & $0.0025-0.0036$ & $0.0031 \pm 0.0004$ & 12.25 & 0.000000142 \\
\hline 2 & Body length & $5.025-5.75$ & $5.4340 \pm 0.2442$ & 4.49 & 0.059632000 \\
\hline 3 & Caput length with mandible & $1.850-2.125$ & $1.9925 \pm 0.0689$ & 3.46 & 0.004743352 \\
\hline 4 & Caput width & $0.85-1.20$ & $0.9450 \pm 0.0826$ & 8.74 & 0.006815823 \\
\hline 5 & Mandible length & $0.825-1.050$ & $0.9388 \pm 0.0582$ & 6.20 & 0.003386518 \\
\hline 6 & Mandible width & $0.225-0.350$ & $0.2750 \pm 0.0356$ & 12.93 & 0.001264179 \\
\hline 7 & Prothorax length & $0.37-0.45$ & $0.4140 \pm 0.0240$ & 5.78 & 0.000572645 \\
\hline 8 & Prothorax width & $0.700-0.825$ & $0.7475 \pm 0.0389$ & 5.20 & 0.001509323 \\
\hline 9 & Metathorax length & $0.26-0.40$ & $0.3223 \pm 0.0384$ & 11.92 & 0.001474944 \\
\hline 10 & Metathorax width & $0.575-0.825$ & $0.7085 \pm 0.0655$ & 9.24 & 0.004287107 \\
\hline 11 & Mesothorax length & $0.3-0.4$ & $0.3260 \pm 0.0274$ & 8.41 & 0.000751582 \\
\hline 12 & Mesothorax width & $0.725-0.875$ & $0.8163 \pm 0.0461$ & 5.65 & 0.002123366 \\
\hline 13 & Abdomen length & $2.125-2.875$ & $2.9400 \pm 0.2248$ & 7.65 & 0.050552576 \\
\hline 14 & Abdomen width & $1.050-1.375$ & $1.2300 \pm 0.0724$ & 5.88 & 0.005236838 \\
\hline 15 & Scape length & $0.080-0.225$ & $0.1328 \pm 0.0349$ & 26.25 & 0.001214383 \\
\hline 16 & Scape width & $0.05-0.09$ & $0.7775 \pm 0.0241$ & 3.09 & 0.000578000 \\
\hline 17 & Pedicel length & $0.05-0.125$ & $0.0793 \pm 0.0142$ & 17.88 & 0.000201000 \\
\hline 18 & Pedicel width & $0.05-0.07$ & $0.0625 \pm 0.0079$ & 12.58 & 0.000061840 \\
\hline 19 & Flagellum length & $1.0-1.4$ & $1.2725 \pm 0.0827$ & 6.50 & 0.006800000 \\
\hline 20 & Flagellum width & $0.07-0.10$ & $0.0810 \pm 0.0055$ & 6.82 & 0.000030530 \\
\hline \multirow{13}{*}{21} & Front legs: & & & & \\
\hline & Coxa length & $0.27-0.32$ & $0.2970 \pm 0.0142$ & 4.77 & 0.00020104 \\
\hline & Coxa width & $0.16-0.2$ & $0.1790 \pm 0.0125$ & 6.99 & 0.00015684 \\
\hline & Trochanter length & $0.14-0.18$ & $0.1655 \pm 0.0119$ & 7.20 & 0.00014184 \\
\hline & Trochanter width & $0.05-0.08$ & $0.0615 \pm 0.0093$ & 15.18 & 0.00008711 \\
\hline & Femur length & $0.73-0.85$ & $0.7835 \pm 0.0345$ & 4.41 & 0.00119237 \\
\hline & Femur width & $0.20-0.26$ & $0.2240 \pm 0.0176$ & 7.85 & 0.00030947 \\
\hline & Tibia length & $0.72-0.80$ & $0.7550 \pm 0.0204$ & 2.70 & 0.00041579 \\
\hline & Tibia width & $0.10-0.17$ & $0.1340 \pm 0.0219$ & 16.31 & 0.00047789 \\
\hline & Tarsus length & $0.2-0.3$ & $0.2610 \pm 0.0308$ & 11.79 & 0.00094632 \\
\hline & Tarsus width & $0.05-0.06$ & $0.0545 \pm 0.0051$ & 9.37 & 0.00002605 \\
\hline & Claw length & $0.09-0.12$ & $0.1045 \pm 0.0083$ & 7.90 & 0.00006816 \\
\hline & Claw width & $0.02-0.03$ & $0.2750 \pm 0.0044$ & 1.62 & 0.00001974 \\
\hline \multirow[t]{13}{*}{22} & Middle legs: & & & & \\
\hline & Coxa length & $0.28-0.33$ & $0.3040 \pm 0.0131$ & 4.32 & 0.00017263 \\
\hline & Coxa width & $0.16-0.22$ & $0.1865 \pm 0.0169$ & 9.09 & 0.00028711 \\
\hline & Trochanter length & $0.15-0.22$ & $0.1890 \pm 0.0205$ & 10.84 & 0.00042000 \\
\hline & Trochanter width & $0.05-0.09$ & $0.0745 \pm 0.0128$ & 17.13 & 0.00016289 \\
\hline & Femur length & $0.65-0.8$ & $0.7370 \pm 0.0456$ & 6.18 & 0.00207474 \\
\hline & Femur width & $0.17-0.23$ & $0.1995 \pm 0.0170$ & 8.52 & 0.00028920 \\
\hline & Tibia length & $0.69-0.76$ & $0.7185 \pm 0.0190$ & 2.64 & 0.00036077 \\
\hline & Tibia width & $0.09-0.013$ & $0.1065 \pm 0.0118$ & 11.10 & 0.00013974 \\
\hline & Tarsus length & $0.22-0.3$ & $0.2590 \pm 0.0229$ & 8.85 & 0.00052528 \\
\hline & Tarsus width & $0.05-0.07$ & $0.0580 \pm 0.0070$ & 11.99 & 0.00004843 \\
\hline & Claw length & $0.08-0.12$ & $0.1030 \pm 0.0126$ & 12.24 & 0.00015894 \\
\hline & Claw width & $0.02-0.03$ & $0.0245 \pm 0.0051$ & 20.83 & 0.00002605 \\
\hline \multirow[t]{7}{*}{23} & Rear legs: & & & & \\
\hline & Coxa length & $0.28-0.33$ & $0.3055 \pm 0.0123$ & 4.04 & 0.00015237 \\
\hline & Coxa width & $0.18-0.22$ & $0.2010 \pm 0.0133$ & 6.63 & 0.00017779 \\
\hline & Trochanter length & $0.16-0.21$ & $0.1875 \pm 0.0133$ & 7.11 & 0.00017764 \\
\hline & Trochanter width & $0.05-0.08$ & $0.0630 \pm 0.0103$ & 16.37 & 0.00010632 \\
\hline & Femur length & $0.90-0.97$ & $0.9100 \pm 0.0243$ & 2.67 & 0.00058947 \\
\hline & Femur width & $0.20-0.26$ & $0.2270 \pm 0.0198$ & 8.71 & 0.00039054 \\
\hline
\end{tabular}


Appendix 4. To be continued

\begin{tabular}{|c|l|c|c|c|c|}
\hline No & \multicolumn{1}{|c|}{ Parameters } & O.R & Mean \pm SD & C.V $(\%)$ & $S^{2}$ \\
\hline & Tibia length & $0.90-0.99$ & $0.9385 \pm 0.0289$ & 3.08 & 0.00083446 \\
\hline & Tibia width & $0.10-0.17$ & $0.1260 \pm 0.0223$ & 17.73 & 0.00049894 \\
\hline & Tarsus length & $0.27-0.32$ & $0.2990 \pm 0.0155$ & 5.19 & 0.00024106 \\
\hline & Tarsus width & $0.04-0.06$ & $0.0545 \pm 0.0069$ & 12.59 & 0.00004710 \\
\hline & Claw length & $0.09-0.15$ & $0.1240 \pm 0.0173$ & 13.94 & 0.00029894 \\
\hline & Claw width & $0.02-0.03$ & $0.0255 \pm 0.0051$ & 20.02 & 0.00002605 \\
\hline
\end{tabular}

